\title{
Usages et limites de la symétrie dans les représentations graphiques en géographie
}

Use and limits of symmetry in graphic representations in geography

Nutzung und Grenzen der Symmetrie in graphischen Abbilshungen in

Geographie

\section{Philippe Martin}

\section{(2) OpenEdition}

Journals

Édition électronique

URL : http://journals.openedition.org/rge/131

DOI : 10.4000/rge.131

ISSN : 2108-6478

Éditeur

Association des géographes de l'Est

Édition imprimée

Date de publication : 1 avril 2007

ISSN : 0035-3213

Référence électronique

Philippe Martin, « Usages et limites de la symétrie dans les représentations graphiques en géographie », Revue Géographique de l'Est [En ligne], vol. 47 / 2 | 2007, mis en ligne le 09 janvier 2009, consulté le 08 septembre 2020. URL : http://journals.openedition.org/rge/131 ; DOI : https://doi.org/ $10.4000 /$ rge. 131

Ce document a été généré automatiquement le 8 septembre 2020.

Tous droits réservés 


\section{Usages et limites de la symétrie dans les représentations graphiques en géographie}

Use and limits of symmetry in graphic representations in geography

Nutzung und Grenzen der Symmetrie in graphischen Abbilshungen in

Geographie

Philippe Martin

\section{NOTE DE L'ÉDITEUR}

Article reçu le 10 juin 2007, accepté après revision le 10 décembre 2007.

\section{1. - Introduction}

1 La symétrie est perçue comme une question tellement banale qu'elle est enseignée dès l'école primaire où l'on fait retrouver, à des enfants de 7 ou 8 ans, les axes de symétrie d'une figure ou d'un dessin. Mais c'est là appréhender de manière réductrice une notion autrement plus fondamentale. D'ailleurs la symétrie, ou plus exactement le concept de symétrie est aujourd'hui fortement sollicité dans la réflexion scientifique, dans une acception plus profonde qu'il n'y parait de prime abord quand, par un amusement fort pédagogique, on fait joindre aux enfants leurs mains devant eux avant de les leur faire ouvrir en faisant jouer une charnière imaginaire située au niveau du petit doigt.

2 Dans les deux approches, l'idée première de symétrie renvoie en fait à celle de régularité, d'harmonie ou d'ordre. Car si tout n'est pas aléatoire, alors le monde est-il peut-être intelligible! Se pose alors la question de savoir comment capturer l'essence de cette complexité symétrique et comment instrumentaliser cette structure ubiquiste 
afin d'accroître notre connaissance sur ce monde? Telles sont bien au fond les interrogations que porte l'idée de symétrie.

Dans quelle mesure cela concerne-t-il alors la géographie ? Rappelons d'une part qu'il n'y a pas de géographie dans un espace homogène, isotrope, sans discontinuités (Brunet, 1968) c'est-à-dire a-symétrique dans la mesure où il présenterait toutes les symétries possibles (un espace brownien par exemple), ce qui revient à n'en posséder aucune. C'est ce que désigne au sens vernaculaire une situation asymétrique. On peut montrer que les brisures de symétries se traduisent par des morphogenèses et donc des morphologies (généralement par la diminution du nombre de symétries) (Delannoy, 1997). En conséquence les formes sont nécessairement en rapport avec la question de la symétrie et ainsi au cœur de la pensée géographique. Comme nous leur avons déjà consacré quelques textes (Martin, 2003 et 2004), nous voudrions ici, seulement, nous focaliser sur la dimension symétrique de ces formes, au sens vernaculaire du terme (symétrie de miroir, symétrie de rotation, etc.), avant d'aborder quelques réflexions sur la fractalité.

4 Cette dimension symétrique peut apparaître dans la caractérisation de l'état de telle ou telle entité, et cela pose la question de la causalité de cette ou de ces symétries, mais cette dimension de symétrie peut aussi être réduite aux représentations, et aux schématisations acquises, et dans ce cas cela pose la question de savoir si cet usage de la symétrie ne fait pas obstacle à une utilisation bien plus théorique de ce concept, qui nécessiterait d'avoir recours à un formalisme $a d$ oc. Une réflexion dialectique entre les symétries habituelles (translation, invariance par rotation par rapport à un point, une droite ou un plan, miroir et la symétrie par dilatation (invariance par changement d'échelle) donc fractale, permettra de poser cette question plus précisément.

5 L'approche fractale nous permet, au moins au niveau des idées, de démontrer l'importance de la symétrie comme structure constitutive des états et des processus spatiaux mono et multi scalaires. Ceci constitue une première ouverture vers une théorie en géographie (Martin et Forriez, à paraître; Forriez et Martin, 2006 et à paraître) ou de la géographie qui intègrera nécessairement cette idée d'invariance d'échelle (rapports constants entre échelles) ou de covariance d'échelle (rapports variables entre échelles, mais suivant une loi spécifique) que recouvre l'idée de symétrie. En conséquence même si un penchant premier nous fait considérer les processus et les manifestations symétriques de l'espace tangible (celui de notre vie quotidienne) comme essentiels, il ne faut surtout pas perdre de vue que cela n'est que l'écume du phénomène, d'un phénomène bien plus profond, et bien plus fondamental.

6 Or il se trouve que la géographie, en étant fort peu théorique, apparaît comme obnubilée par le rapport entre perception et représentation. En conséquence il ne faudrait pas que ce tropisme fort peu scientifique au sens des sciences dures (falsifiables) masque ou limite la possibilité qu'a cette discipline depuis peu de s'établir comme une connaissance largement théorique qui se donnerait comme horizon d'expliciter (et non de représenter) les raisons des manifestations spatiales et scalaires de l'espace tangible qu'elle analyse depuis longtemps par des méthodes inductives, voire hypothético-déductives. Il est classique de considérer en géographie qu'une représentation, si elle n'est pas une explication au sens fort du terme, n'en est pas moins fortement explicative. Il nous semble que sous cette dialectique qui s'appuie grandement sur des pratiques didactiques - la géographie est aussi une discipline 
scolaire - se cache une certaine confusion sur laquelle il convient de dire deux mots afin que nos propos ne soient pas mal compris.

7 Dire qu'une représentation n'explique rien, c'est au fond énoncer un truisme. Ainsi au plan lexical si deux mots existent on peut en inférer qu'ils ne recouvrent pas le même sens. D'une certaine façon la représentation ne fait "que » présenter; c'est-à-dire mettre dans un format accessible à une intelligence une situation, mais sans que cette mise en forme ne soit totalement contrôlée. En d'autres termes deux auteurs pourront arriver à deux représentations différentes d'égale valeur. Expliquer c'est au contraire fonder une compréhension sur des principes premiers; c'est donc construire une théorie totalement contrôlée et contrôlable (falsifiable), ce qui demande des concepts exprimés dans un formalisme $a d$ oc, sans lesquels les phénomènes eux-mêmes, bien qu'ils puissent être dans une représentation ne sont pas observables. C'est par exemple le cas des formes fractales. Combien de géographes ont tracé des courbes fractales sans savoir qu'ils utilisaient une symétrie par dilatation! Tous les géomorphologues ont observé des reliefs sans trop rapprocher leurs irrégularités de détail à la forme d'ensemble, mais sans établir de rapports entre les échelles d'irrégularités, L'essentiel de l'explication comme telle réside donc dans l'enchaînement formel qui contraint la pensée afin de rendre compte (de formaliser) d'une seule façon des régularités du monde. Ainsi n'y a-t-il qu'une équation qui relie la matière à l'énergie. C'est dans cette perspective de constitution d'un savoir à la fois partagé et unique que la symétrie est appelée à jouer un rôle central dans une expression scientifique, en particulier en géographie.

8 Etant un outil qui dit l'invariance dans différentes situations, donc quel que soit le substrat, et indépendamment de la discipline, son expression ne peut être que mathématique, car nécessairement formelle et abstraite. Ainsi établi, cet outil peut être utilisé en physique en particulier en raison de la proximité formelle de ces deux disciplines, mais aussi en biologie ou en géographie, La preuve en est que l'on peut définir mathématiquement des opérations de symétrie, des groupes de symétrie, des symétries affines, d'autres qui ne le sont pas, etc., soit autant de notions et de possibilités fondamentales qui sont à la racine des manifestations tangibles, y compris biologiques et anthropiques. A la question d'Emile Noël: "Qu'avez-vous envie de proposer [aux biologistes] ?» R. Thom répond au sujet de la théorie des catastrophes : "Je leur propose une formalisation de l'embryologie par des entités abstraites. Il n'y a de science que dans la mesure où l'on plonge le réel dans un virtuel contrôlé ».1 C'est ce qu'il est peut-être possible de faire aujourd'hui en instrumentalisant la notion de symétrie qui apparaît comme une des composantes de ce virtuel contrôlé dans lequel nos problèmes spatiaux et scalaires devront être plongés.

9 Il découle de tout cela que cette réflexion liminaire sur la symétrie est un préalable scientifique et épistémologique au développement d'une théorie et peut-être plus, à sa diffusion. Il n'est donc pas sans signification qu'il ait fallu attendre aussi longtemps pour voir aboutir un premier numéro thématique de revue autour de ces questions. Dans le cadre des contraintes formelles de cet article, elle ne peut qu'être trop brièvement esquissée, mais sera développée ailleurs. Il n'est d'ailleurs pas sans signification qu'il ait fallu attendre aussi longtemps pour voir aboutir un premier numéro thématique de revue autour de ces questions. 


\section{2. - Des formes symétriques?}

10 Avec sa symétrie d'ordre neuf, avec son plan radial, avec ses fortifications, la ville renaissance de Palma Nuova (Italie) illustre assez bien l'idée de symétrie d'une forme dans l'espace tangible. On y perçoit une fortification de forme classique, «à la Vauban ", mais déployée sur un terrain qui ne contraint pas l'ouvrage de protection en fournissant des points d'appui utilisables dans une stratégie défensive ce qui la conduirait à lui faire perdre sa symétrie ou une partie de ses symétries. Sur la figure $n^{\circ} 1$, on notera la place des portes au nord-ouest, au nord-est et au sud par lesquelles il est possible de faire passer chaque fois un axe de symétrie. A cela s'ajoute bien sûr une symétrie par rotation d'ordre 9. D'autres éléments sont encore remarquables comme la disposition en cercles concentriques (donc en échelle) des bâtiments.

Figure 1 : Plan de la ville renaissance de Palma Nuova (Italie).

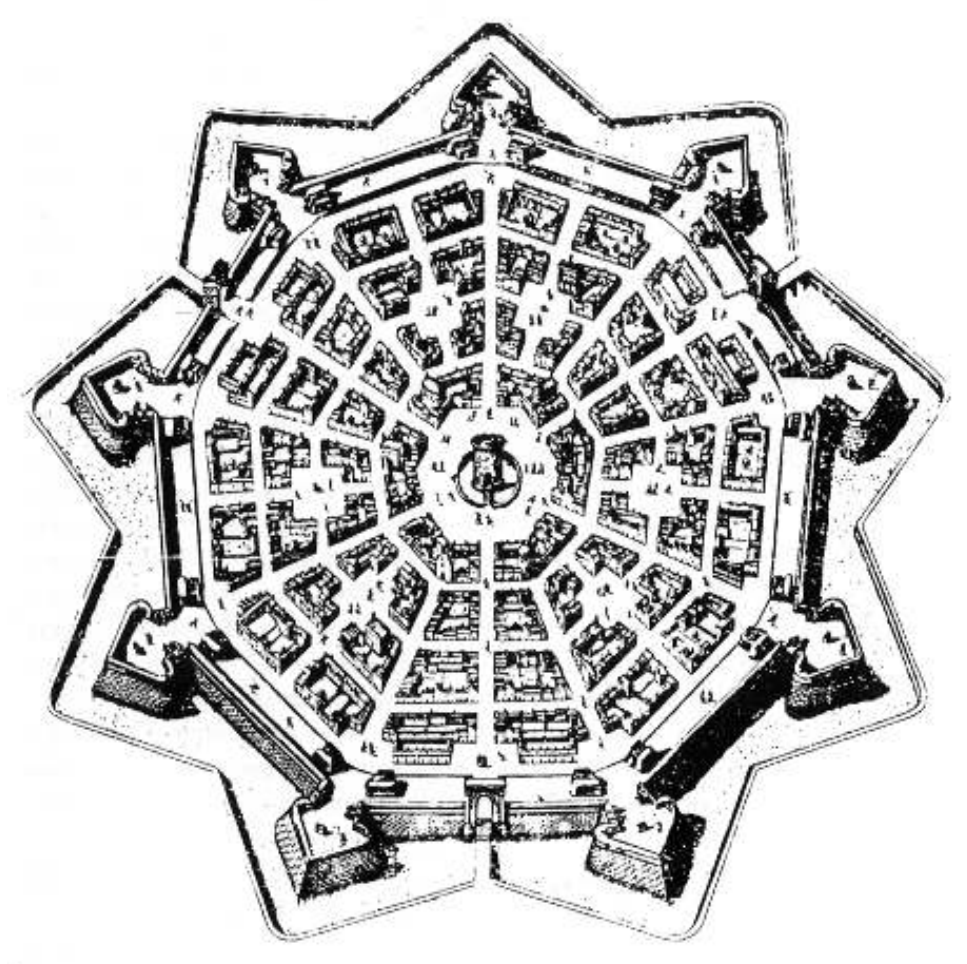

Nous pouvons en outre ici faire une remarque d'ordre plus général dans la mesure où l'importance des symétries partielles d'un objet est fonction des contraintes de son milieu. Cela est vrai pour ces fortifications comme pour des objets naturels. Ainsi la terre qui est globalement une sphère le doit à la symétrie des forces qui s'exercent sur elle comme le fait remarquer G. Lochak (Lochak, 1994, p. 16): "[pour expliquer la rotondité de la terre, Aristote] s'appuie sur le peu qu'il devine, à savoir une loi générale de symétrie: en somme pour lui, la Terre est sphérique parce que la loi de la gravitation doit être isotrope ». 


\section{1. - La symétrie, quelles clefs de lecture?}

Il est clair que les symétries classiques (translation, rotation, en miroir, etc.; self similaire ou affine) renvoient aux modalités d'ordre statique existant, pour ce qui nous concerne, dans l'interface terrestre. Mais avant toute chose, il faut bien voir que cette idée correspond à une modalité fondamentale d'organisation qui rend intelligible les structures, les règles, les configurations spatiales qui l'utilisent. L'approche doit donc être dialectique et il est peut-être possible de rassembler les différentes implications pratiques du concept de symétrie (Tableau 1). Cela étant, il ne faut pas chercher dans ce tableau des correspondances en ligne, dans la mesure où les aspects développés dans les colonnes 2, 3 et 4 peuvent être rapportés à chacun des cas de symétrie donné dans la colonne 1. Inversement il est possible d'envisager des symétries dans le temps, dans l'espace classique (2D, 3D) et dans l'ordre des échelles. La symétrie transcende donc la condition humaine (colonne 2) ce qui ne veut pas dire que l'homme n'ait pas instrumentalisé certaines symétries et donc ne les ait pas transmises préférentiellement (cf. l'usage des symétries et l'approche euclidienne du monde).

Dans cette perspective le rapport de la symétrie à l'espace est essentiel. C'est bien sûr l'espace contenant où s'expriment des symétries basiques et très tangibles (translation qui conduit au pavage par exemple), mais c'est aussi l'espace comme construction abstraite et ensemble de règles qui interfère avec l'idée de symétrie, qui intègre cette idée.

\section{2. - -Des formes anthropiques symétriques?}

C'est aujourd'hui un truisme que d'affirmer que beaucoup de formes anthropiques sont symétriques parce qu'elles correspondent à des formes euclidiennes ou à des formes composées à partir de figures euclidiennes. Nous avons vu ci-dessus le cas d'une place forte. Les multiples gratte-ciel portés par leur course au gigantisme, à grande hauteur, relèvent aussi de cette logique. Difficile d'introduire de trop grandes irrégularités sans approcher les limites mécaniques des poutrelles en acier ou en béton, plus ou moins précontraint. Certes l'idée n'est pas neuve et déjà l'Egypte antique avec ses pyramides, d'abord à gradins puis lisses, en est un exemple fondateur. Les Grecs ensuite qui ont déployé un fronton dit grec, en utilisant le nombre d'or. Rappelons enfin que souvent un plan d'eau sert de miroir à un édifice comme dans le cas du Taj Mahal en Inde ou dans celui des châteaux de Chenonceau et d'Azay le Rideau en France. Cette question traverse donc l'histoire de l'humanité, bien que toutes les époques et tous les lieux ne soient pas affectés de la même façon, même si la symétrie est moins présente, semble-til, lorsque les pouvoirs, en termes de prégnance plus que de capacités économiques ou techniques, sont plus faibles. 
Figure 2 : Vase ou visages ; que percevons-nous?

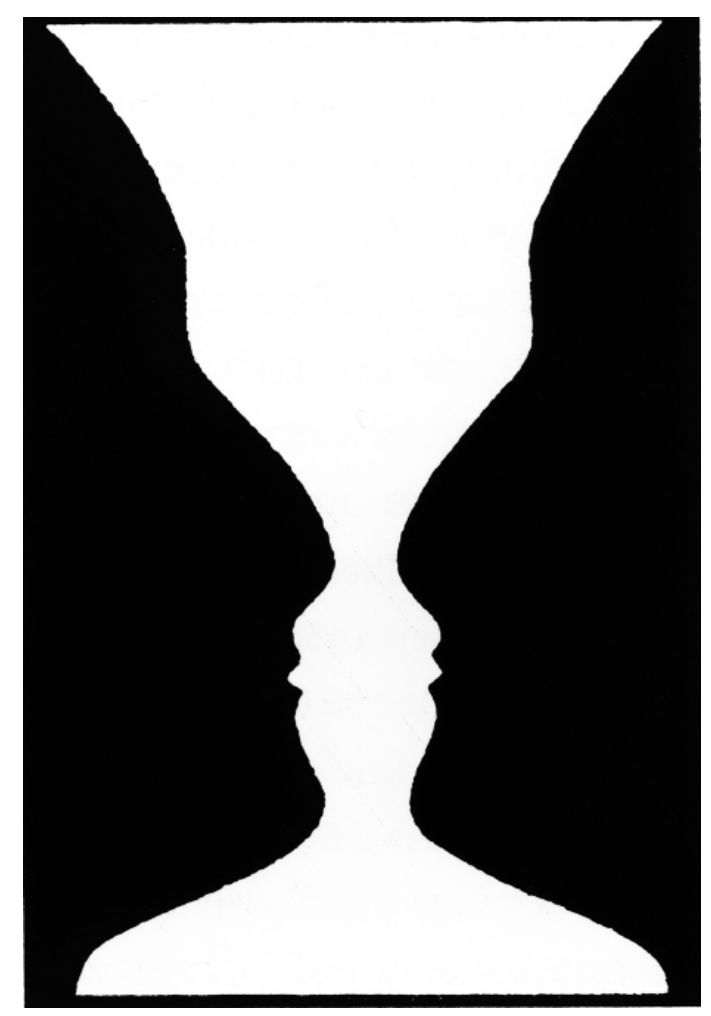

15 Pourquoi l'homme bâtit-il souvent en utilisant des figures euclidiennes qui manifestent des symétries apparentes à l'échelle de l'ouvrage? La question vient de loin mais il semble que l'on ne puisse dissocier la construction de la géométrie euclidienne d'un arrière fond relatif à la perception de - à l'appétence pour - la symétrie, perception peut-être influencée par tout ce que tout le monde peut noter à savoir que le corps humain (mais aussi de bon nombre d'animaux) est symétrique (symétrie bilatérale), du moins apparemment et dans une certaine mesure. Le côté droit n'étant pas identique au côté gauche et certains organes comme le cœur étant uniques à la différence des reins ou des poumons. Par ailleurs, la question de l'émergence de la géométrie euclidienne (des formes euclidiennes) renvoie semble-t-il à celle de l'arpentage dans l'Egypte antique des champs situés dans le lit majeur du Nil dont les tailles, les surfaces devaient être définies afin d'organiser la production et afin de pouvoir prélever l'impôt. Initiant peut-être là la question, toujours d'actualité, des bases fiscales. C'est donc au fond d'une question de pavage non lacunaire qu'il s'agit et l'on sait combien la translation de quelques figures euclidiennes peut y concourir!

\subsection{1. - -Des formes anthropiques essentiellement euclidiennes}

16 Pourquoi bon nombre de réalisations anthropiques, des bâtiments par exemple mais pas seulement, utilisent-elles particulièrement certaines symétries en rapport avec la géométrie euclidienne alors que la nature montre qu'elle est, dans sa grande majorité, fractale ; c'est-à-dire qu'elle met en œuvre une autre forme de symétrie, une symétrie qui se déploie dans l'ordre des échelles, une symétrie qui met en œuvre un processus de dilatation (ou de contraction) ? Cette question soulève bien sûr celle des capacités du cerveau humain et des apprentissages culturels qui confèrent une grande aptitude à penser l'angle droit, la droite ou le plan orthoclinal et une grande difficulté à penser ce 
qui est irrégulier, donc fractal, et développé dans l'ordre des échelles. Cette dimension reste à conquérir, même pour les géographes, et l'on n'a pas, nous semble-t-il, bien mesuré toute l'importante de ce type de symétrie.

Mais si les formes naturelles ne sont pas en général euclidiennes, elles ne sont généralement pas lisses non plus. L'irrégularité, la rugosité sont la marque d'une large part des morphologies de la nature, surtout dans le monde des sciences naturelles. Ainsi n'importe quel paysage naturel ou vu dans la nature exhibe-t-il une irrégularité qui souvent en fait son charme. A partir de là, en première approximation, il est peu probable que l'idée de copier la nature dans ses formes ait été aussi forte que certains veulent bien le dire. En tout état de cause ce n'est pas l'exemple le plus courant qui a guidé les choix architecturaux, occidentaux en particulier. De même les constructions mathématiques de l'antiquité ne renvoient guère à des exemples tangibles réellement observables; les évolutions stellaires étant beaucoup plus conçues et reconstruites que vues. Il y a donc là toute une réflexion à conduire, que nous ne pouvons qu'effleurer, en envisageant quelques hypothèses. L'homme se serait-il posé en s'opposant? La rareté d'objets très symétriques au sens courant comme les cristaux l'a-t-il inspiré ? Les formes euclidiennes: carré, pyramide, cône, sphère, etc. présentant une grande stabilité, elle-même liée à leurs symétries, auraient été choisies pour cette raison ? Leur caractère mono scalaire, voire presque a-scalaire à un niveau conceptuel, les rend peutêtre plus appréhendables?

Le choix de formes essentiellement euclidiennes pourrait donc être vu, soit comme un positionnement des hommes contre l'organisation habituelle de la nature - cela lui permettant de se poser en s'opposant - soit (et) comme la résultante de capacités physiologiques particulières, elles-mêmes liées à un prégnance de la symétrie sous la forme d'un principe fondamental ou essentiel de l'univers. Certaines réalisations, comme par exemple les pyramides d'Egypte, ou le château de Versailles, laissent penser qu'il y a là un processus anthropique finalisé, qui instrumentalise la symétrie (cours carré du Louvre, Arche de la Défense, etc.), par lequel l'homme, ou les plus dominants d'entre eux, étalonne(nt) sa (leur) puissance. Même si cela est vrai, ce n'est toutefois qu'une partie de la réponse.

19 Ces formes présentant le plus souvent plusieurs axes de symétrie, n'en ont pas moins généralement des surfaces lisses, régulières, "propres ", ce qui est parfaitement évident avec les immeubles de grande hauteur aux parois de verre ou avec certaines sculptures de Brancusi. Il y aurait donc comme une fascination du lisse (cf. le rôle du marbre dans l'architecture monumentale avant l'avènement du verre), du poli et une aversion pour le rugueux, pour ce qui est (perçu?) comme inachevé. La langue française, plus ou moins argotique, est riche d'expressions qui disent cela. Ne parle-ton pas de situations carrées, propres, nickels, etc. ; soit autant de formulations imagées qui disent la fascination de la symétrie, du poli, du lisse. Ce qui nous importera donc dans les réflexions qui suivent, c'est de savoir quel peut être le poids de telles structures anthropologiques, d'une part dans la conception et dans la représentation que tout un chacun se fait de situations géographiques diverses et, d'autre part d'évaluer quel peut être le niveau de prégnance de ce mode de pensée dans la définition et l'emploi des chorèmes et de la chrorématique. En d'autres termes ces derniers sontils plus la marque de structures anthropologiques, psychologiques, etc. fort actives chez chacun d'entre nous ou sont-ils un réel moyen d'épuration, de clarification des structures et des dynamiques spatiales de l'espace tangible? La validité de cette 
approche dépend de la réponse apportée à cette question mais le problème essentiel est de savoir de quoi faire dépendre la validité de la réponse.

Il faut toutefois considérer que ces conceptions présentées sont manifestement celles d'un monde plutôt matériel et/ou matérialiste mono-scalaire qui s'estompe (Martin, 2004). Mais il nous semble que ces questionnements très généraux ont une résonance évidente avec certaines pratiques géographiques comme la chorématique ou la schématisation (voire la cartographie) dont la représentation finale doit être, par construction, logiquement close, achevée, "propre ", sans aspérité, lisse en quelque sorte. Ceci débouchant, et c'est là tout l'intérêt d'aborder ce problème, sur la question des conditions de la pensée géographique en particulier ou peut-être sur celle des contraintes s'exerçant sur la pensée en général et géographique en particulier, sur ce que nous avons évoqué ailleurs par le terme d'obstruction (Martin, 2006.a) relevant d'une impossibilité, pour des raisons multiples, de penser (ou de faire) autrement. Il est clair que toute production scientifique doit pouvoir dépasser ces situations plus ou moins bloquées et l'on peut faire l'hypothèse que cela doit mieux se produire si les conditions de la pensée existante sont finement analysées. Il nous semble que le faible entrain en géographie pour une approche théorique, pour une théorie de l'espace géographique relève de ces logiques.

\section{3. - -La forme comme morphologie tout autant que comme structure spatiale}

21 Pour aller au-delà d'une description phénoménologique des formes nous pouvons premièrement noter qu'à chaque réitération d'une situation de non équilibre, comme avec les cellules de Bénard par exemple, la même organisation spatiale apparaît (en boucle ou en cercle). Nous sommes donc amenés à considérer qu'il existe, en arrière plan, une structure abstraite qui doit pouvoir être exprimée mathématiquement dans l'espace tangible mais aussi dans un espace abstrait celui des attracteurs. Les archétypes des formes parmi lesquels on trouve : la spirale, la courbe de flambage, etc. (Martin, 2004) peuvent être considérés comme des états limites stables, au même titre que la configuration d'un balancier immobilisé au-dessus d'un point situé sur sa verticale. Ce point est un lieu de l'attracteur du système dynamique de la pendule correspondant à un état particulier dont l'horloge ne sortira que par injection d'énergie (si on « remonte» la pendule). Il en est de même pour les formes archétypales. Ceci a d'ailleurs était noté par les géomorphologues depuis longtemps.

Toutefois le faible nombre de configurations de base (Martin, 2004, p. 28) peut aussi expliquer que des causes efficientes très différentes, dans le cadre d'une indépendance entre forme et substrat, conduisent à des morphologies très semblables (cf. la courbe de flambage que l'on retrouve dans les méandres et dans les ondulations des serpents). C'est le classique phénomène de convergence des formes.

Nous pouvons deuxièmement rappeler qu'expérimentalement, il a été démontré, soit par des algorithmes aléatoires, soit par des expériences physiques, soit enfin par des mesures sur des objets naturels, que l'établissement de réseaux de drainage de forme arborescente relève d'une dynamique qui est sous la dépendance directe des propriétés générales de l'espace. Ainsi si un réseau (hydrographique par exemple) doit couvrir ou remplir un espace il faut qu'il se déploie en respectant le principe d'économie. La structure arborescente assurant ces cheminements courts et directs (Stevens, 1978). Sa 
croissance s'effectuant en périphérie par l'établissement de petites branches. Cette forme est récurrente, mais, toutes choses étant égales par ailleurs, ni la disposition dans l'espace de chacune des branches ni leur nombre exact, ne sont identiques. C'est l'effet du hasard. L'une des possibilités de cette économie de moyen est de mettre en œuvre le principe de symétrie. Celui-ci permet de déplacer et de répliquer une même forme qui dans le cas hydrographique ressort des convergences aléatoires de filets d'eau fixés par glyptogenèse. Les formes fractales ne sont de ce point de vue que des formes répliquées par dilatation. La question est donc toujours de savoir -1- si une structure abstraite contrôle le déroulement de la morphogenèse - celle-ci ne permettant qu'un ensemble de solutions - et -2- quel rôle joue la symétrie dans cette structure abstraite?

Figure 3 : Voir un triangle invisible.
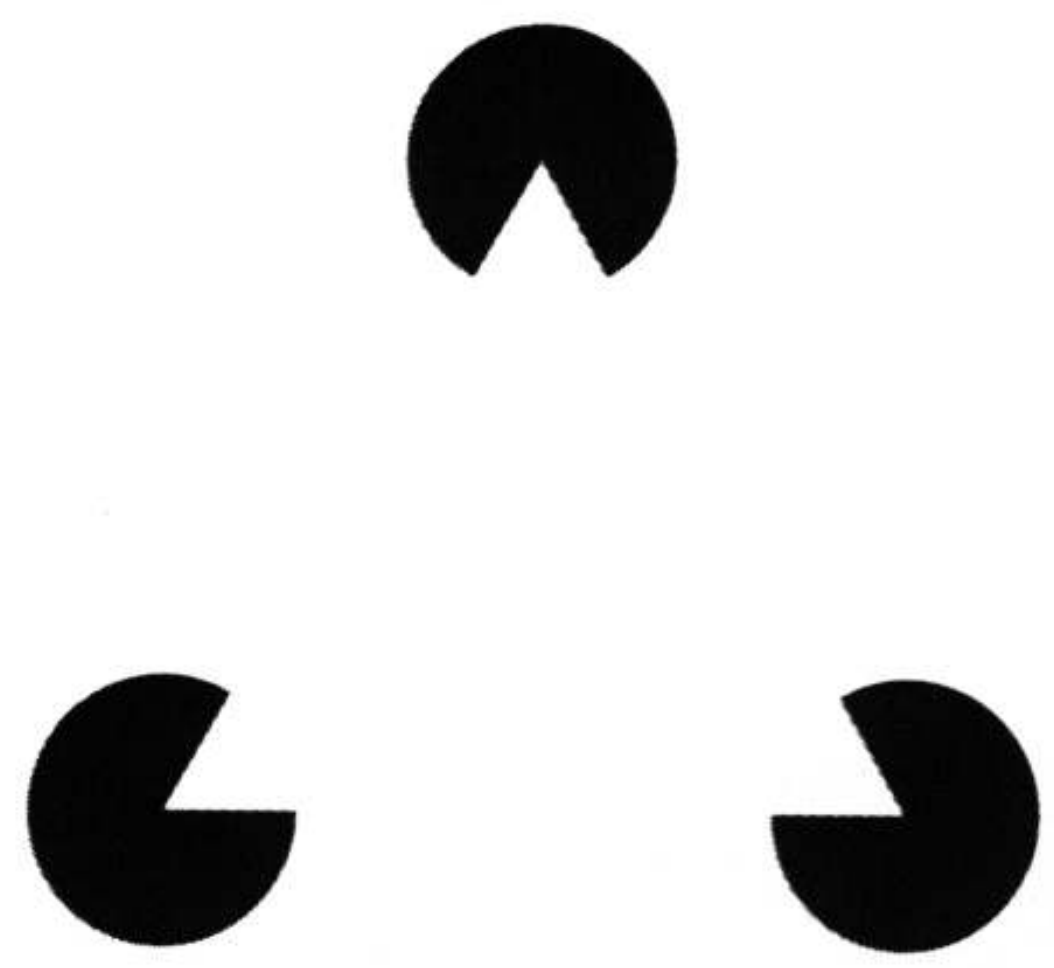

Pour prolonger cette analyse, nous pouvons partir d'une autre constatation. Une très large partie des formes observées sur l'interface terrestre sont des fractales du moins dans la limite d'échelles dites de coupure. Si de telles formes sont plus abondantes que des formes euclidiennes cela signifie aussi que la géométrie qui les décrit est plus générale que la géométrie euclidienne. C'est ce que constate aussi L. Nottale: "Les géométries fractales sont simplement plus générales que les géométries euclidiennes et courbes" (Nottale, 1998, p. 181). Reste à formaliser une explication de cette ubiquité peut-être en utilisant le principe de moindre action. Ces trois éléments: le rapport entre archétypes et point stable d'un attracteur, la symétrie par dilatation comme la manifestation d'un principe d'économie et le très grand champ d'application de la géométrie fractale, dont aucun ne relève d'une stricte perception mais plutôt d'une approche théorique constructiviste, montrent que la seule prise en compte phénoménologique des formes est très insuffisante et qu'il convient de développer une théorie des formes et/ou des configurations spatiales. 


\subsection{1. - Symétrie et description de la forme}

anthropiques et le calcul de sa ou des ses dimension(s) fractale(s) sont les seules méthodes actuellement disponibles pour faire émerger le schéma de base de l'organisation d'une forme irrégulière. C'est là un point fondamental et justifié. Justifié car il permet de qualifier la forme et de comparer les formes entre elles. Fondamental car il touche à l'essence même de la forme sans exclure les échelles les plus grandes ou les plus petites, sans obligatoirement introduire des coupures mal ou peu justifiées, ou des échelles dites significatives, mais de quoi ? La description fractale est donc une description qui est essentielle car minimale et plus globale et donc plus fondamentale que la description naturaliste pratiquée jusqu'alors. Ceci renvoie en outre à une autre dimension qui est d'axer notre approche sur une étude des structures beaucoup plus que sur des trajectoires individuelles, sur les règles fondamentales beaucoup plus que sur les cas particuliers. Cela revient à avoir une approche structurelle, sur le modèle historique de Cl. Levi-Strauss, dont dès $1973 \mathrm{R}$. Thom (Thom, 1973, 2003) soulignait qu'elle est la seule possible en particulier en science sociale: «Les scientifiques doivent se convaincre que l'approche réductionniste est en général trop ambitieuse et doit céder la place à l'approche structurale, infiniment plus modeste dans ses buts et ses méthodes. (...). L'approche structurale a pour but de simplifier la description en donnant un nombre fini de règles combinatoires portant sur des morphologies élémentaires qui permettent de reconstruire toute morphologie empirique. Il s'agit en gros, d'éliminer de la description le plus d'arbitraire possible, en mettant en évidence ses symétries implicites, son organisation cachée. » Ainsi s'il n'est pas admissible de postuler une finalité dans un discours scientifique, rien, au contraire, n'empêche de penser qu'une certaine causalité formelle est à l'œuvre dans la nature. Nous n'en prendrons pour preuve à ce stade que les principes de symétrie, de conservation, de moindre action, qui peuvent être considérés comme des causes formelles.

28 A partir d'une telle posture il est possible de s'interroger sur les moyens classiques, cartographiques de descriptions et en particulier, pour ce qui est du relief, il est logique d'envisager le rôle qu'a joué et que peut jouer, par exemple, la carte géomorphologique. Comment représenter - faut-il même le faire - des morphologies fractales? La 
question se pose aussi pour des configurations anthropiques. Les chorèmes ont été une forme de réponse dont on perçoit qu'elle est très structurelle du moins si l'on s'en tient à la définition programmatique de $\mathrm{R}$. Thom donnée ci-dessus. Mais la question qui vient après est de savoir si le cerveau comme outil unique de compréhension, voire même le groupe comme ensemble produisant la réflexion, est susceptible de proposer une connaissance aussi achevée que cela est souhaitable sans les "béquilles" que représentent les formulations théoriques et les tests effectués contre la théorie?

\section{4. - Symétrie et représentation}

L'objectif est ici d'abord de nous interroger sur ce que nous percevons avant de considérer comment certains éléments de l'espace géographique sont représentés. Rappelons que nous ne percevons qu'une partie et peut-être même qu'une petite partie de la symétrie du monde.

\subsection{1. - Que voyons-nous?}

Quand nous percevons que percevons-nous? Comment structurons-nous notre perception? Sur la base de lignes, de limites, de discontinuités, assurément mais pas seulement. Dans un souci de simplification nous nous limiterons à la vue laissant de côté les autres sens. Ce qui permet de voir c'est par exemple la différence entre un fond et une forme, c'est la limite entre deux domaines. Mais ce qui permet de «voir » aussi c'est de pouvoir reconstruire un objet signifiant à partir de structures connues, d'ordres possibles dans lesquels la symétrie joue un rôle essentiel. En cela elle joue un rôle de guide et de moyen de compréhension, d'accès à l'intelligibilité du monde. Ainsi la prise de conscience de ce que nous voyons peut être décrite comme l'émergence d'une discontinuité, comme un basculement de type catastrophique au sens thomien du terme (Thom, 1977, 2003, Stewart, 1982), c'est-à-dire comme une rupture de symétrie qui va être créatrice d'un nouvel ordre mental de connaissance.

\subsubsection{1. - Vase ou visage?}

Que voyons-nous dans la figure $\mathrm{n}^{\circ} 2$ où le fond et la forme peuvent être alternativement blanc (che) et noir(e). Ce qui permet de "voir » c'est-à-dire d'avoir une compréhension de l'image, c'est certes la limite entre les deux domaines mais pas seulement. Ce qui permet de voir et de douter ce sont les formes de référence mémorisées (vase, visage) et les symétries; symétrie du vase par rapport à son axe vertical ou symétrie en miroir des visages? C'est donc la concordance topologique de deux axes de symétrie qui crée la confusion mais aussi l'intérêt du dessin.

L'une ou l'autre forme se révèle par la prise de conscience d'un assemblage cohérent de limites. La logique de la symétrie y contribuant largement.

\subsection{2. - La perception d'un triangle illusoire}

La figure $\mathrm{n}^{\circ} 3$ montre un triangle bien que celui-ci soit non figuré. Le triangle n'est visible que parce que ses limites sont suggérées par l'orientation des portions triangulaires retirées des disques noirs. Il suffirait que l'un des disques soit tourné autrement pour que le triangle «n'apparaisse » plus. Une forme perçue est donc une 
question de topologie, de positionnement dans l'espace mais elle est aussi la résultante des reconstructions que l'esprit peut réaliser. Dans ce cas là aussi la symétrie permet de «lire» la figure et de produire du sens. Il est ainsi aisé mentalement d'élever les bissectrices et de trouver le barycentre, c'est-à-dire le point d'équilibre de l'ensemble.

Autrement dit les structures mentales dont nous disposons sont évidemment essentielles dans la compréhension de ce que nous observons et dans ces schèmes, il y a celui de la symétrie. Dans ces conditions il n'est pas trop étonnant qu'elle soit utilisée pour des représentations géographiques. Mais quand nous faisons cela est-ce que nous projetons des structures mentales ou est-ce que nous représentons les structures réelles de l'entité observée?

Figure 4 : L'hexagone français : combien d'axes de symétrie (d'après R. Brunet, 1973) ?

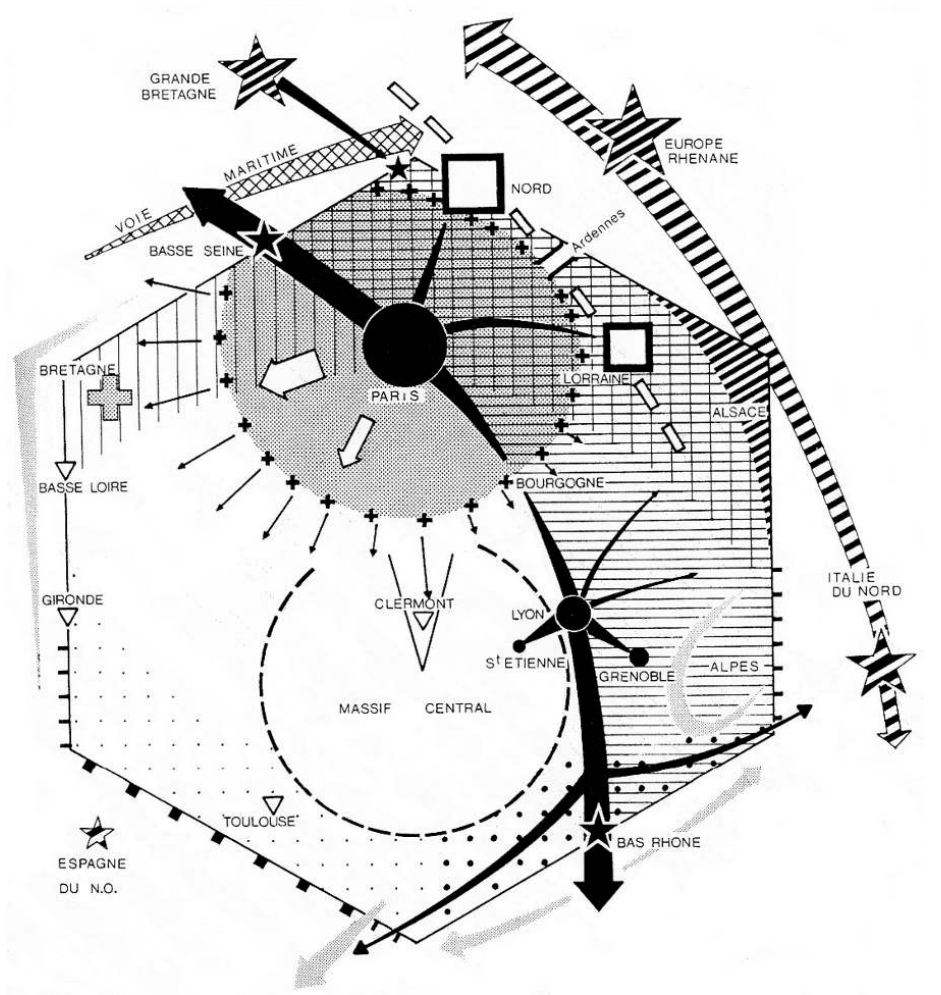

En géographie, au travers des chorèmes en particulier mais pas seulement - pensons à la figure imposée qu'est l'hexagone et qui est bien antérieure mais portée par la même volonté didactique - la symétrie occupe une place prépondérante dans les modes de représentation. On pourrait même dire que réaliser une représentation géographique conduit peu ou prou à mettre en œuvre la symétrie qui agit comme un guide, comme un filtre. Cela est évident lorsque l'on considère le bestiaire des signes et autres trames que nous utilisons, mais cela ne l'est pas moins à plus petite échelle lorsque nous structurons un schéma par exemple. Il faudrait en outre se questionner sur l'usage de la symétrie pour traduire certaines représentations intellectuelles du monde afin d'en donner une image plus ordonnée, ou ordonnée différemment de ce qu'il est, comme cela peut être le cas avec la régularisation des structures fractales, des morphologies irrégulières. En changeant de symétries, en troquant un objet à symétrie d'échelle contre un objet régularisé, lisse, euclidien que faisons-nous, que perdons-nous ? Reste que subrepticement nous passons d'une invariance consubstantielle à la matière et au 
formalisme qui peut la décrire, à une symétrie qui est perçue et projetée et qui est donc de l'ordre de la représentation. Ainsi en géographie les chorèmes, ces idées figurées de forces, ou de contraintes, sont-ils particulièrement symétriques et assemblés souvent en structurant la représentation réalisée à petite échelle en fonction d'une symétrie plus ou moins perceptible dans le territoire. C'est, pourrait-on dire, une modalité chrono didactique de représentation qui est induite par une posture scientifique qui considère préférentiellement les conséquences visibles des processus apparents pensés comme déterminants dans une analyse très largement socio économique.

\subsection{3. - Les chorèmes et la chorématique}

"Les chorèmes sont des structures élémentaires de l'espace" selon la formule de R. Brunet (Brunet, 2001) qui, dans son célèbre tableau a placé « en ligne les principales actions des sociétés " et "en colonne les éléments de base pour une représentation iconique ". La mise en œuvre correspondant à une modélisation graphique qui permet de saisir les grands traits d'une situation, d'une configuration, voire son évolution. Ces chorèmes essayent donc de rendre compte d'une complexité avérée par une représentation compliquée en se fondant

- d'une part sur des a priori idéologiques, en particulier économiques (force économique, potentiel, barrière, etc.), tout en reprenant un vocabulaire qui est plus celui d'une physique du XIX ${ }^{e}$ siècle qu'autre chose ; c'est au fond un essai de formalisation graphique qui se heurte - mais pouvait-on (peut-on encore) faire autrement ? - au déficit théorique de la géographie (Hubert, 1993);

- et d'autre part sur des apprentissages classiques (géométrie euclidienne, cartésianisme, etc.) qui conduisent à penser le monde comme étant lisse et régi par des forces, économiques en particulier, mais pas seulement (cf. les logiques de blocs, le choc des civilisations, etc.), opposées.

La symétrie des constructions découle largement de cela, de ce rapport: force/ résistance. Cela étant, souvent la représentation montre une dissymétrie (soit un écart à un niveau imaginé de symétrie pensé comme nécessairement abouti), c'est-à-dire une symétrie qui n'est pas aussi parfaite qu'elle aurait pu l'être ou que l'on aurait voulu qu'elle soit. 
Figure 5 : Cartographie schématisée du détroit du Pas de Calais (d'après B. Picouet

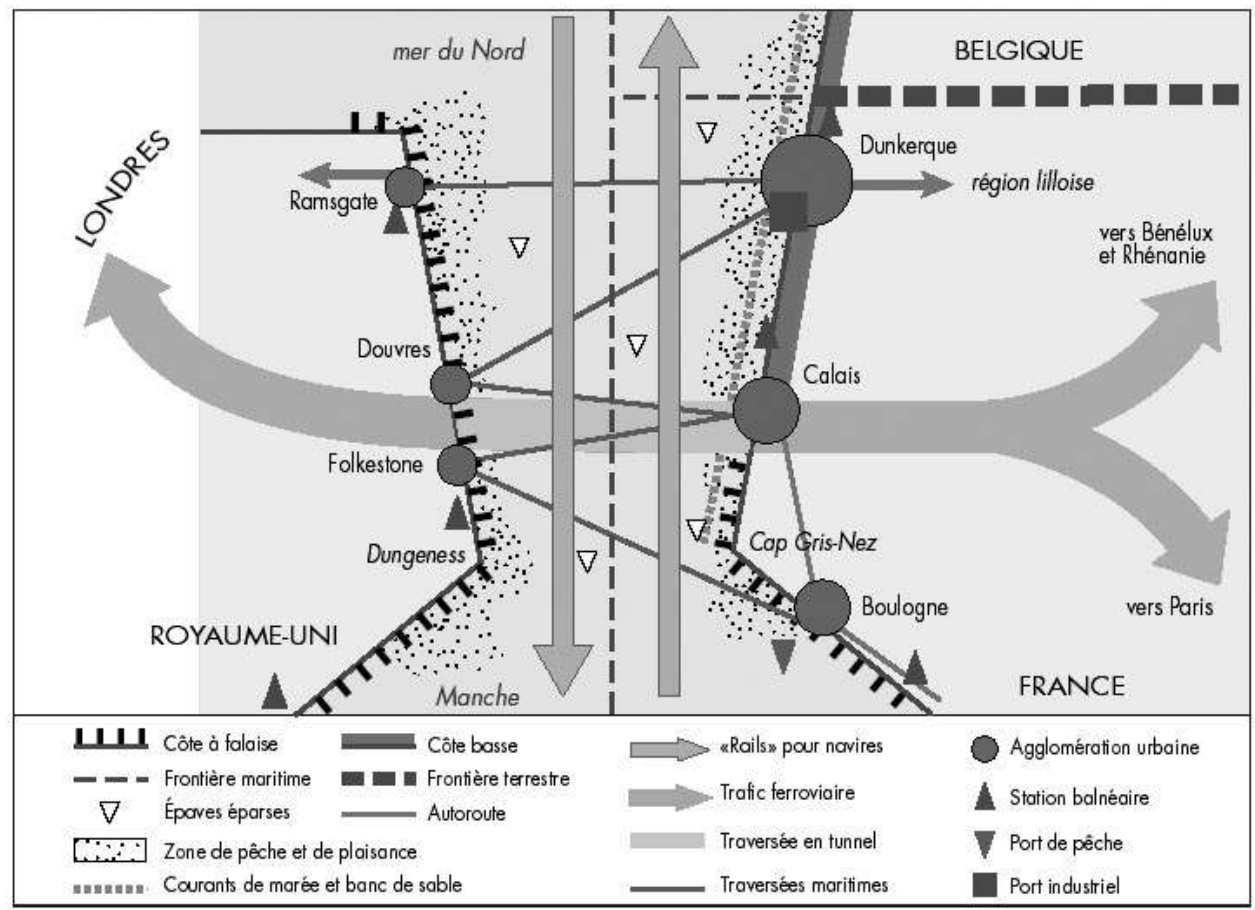

Il faut toutefois noter que si l'on peut parler de "structures élémentaires de l'espace " et si celui-ci est entendu dans un sens abstrait (attracteur) et non pas ramené à un simple contenant, ces structures devraient plutôt être : la symétrie et l'échelle et non le point, la ligne, l'aire, etc. qui ne sont que des concepts euclidiens statiques qui, pour partie, traduisent le besoin d'aborder cette vaste et profonde question du passage du continu au discontinu dans l'espace tangible. Ce dernier pour une part se comprend maintenant (Nottale, 1993) en formalisant le problème en termes fractals (covariance). Apparaît ensuite la question de la dynamique, de la nécessaire dégradation de l'énergie indispensable à toute morphogenèse qu'elle soit naturelle ou anthropique (Bois, 2002); c'est en partie ce dont rend compte la seconde dimension de la matrice de R. Brunet (Brunet, 2001, p. 198). L'intérêt des chorèmes est certainement dans leurs apports topologiques mais du point de vue de la complexité au sens strict, c'est une démarche discutable qui toutefois semble bien en accord avec une pensée française décrite souvent comme très (trop ?) cartésienne ce que tend à prouver la prégnance du fameux hexagone français.

La représentation qu'en donne en 1973 R. Brunet (Figure 4) est fondée sur l'idée de construire un modèle graphique dont on voit les prémisses dans un article de 1972, par épurations successives, de façon à arriver à l'essentiel qui largement a trait au réseau de villes. Le résultat montre que dans une telle représentation la symétrie classique est partout, ce qui conduit à se demander si réellement le territoire est structuré de cette façon? Outre l'hexagone qui est remarquablement symétrique nous avons la duplication (translation), de l'axe essentiel du pays : Basse Seine-Paris - Lyon-vallée du Rhône, avec la figuration de celui qui joint l'Europe rhénane à l'Italie du nord. Par ailleurs la moitié sud - ouest du territoire métropolitain "vide " s'oppose à la moitié nord - est pleine tout comme un large bassin parisien «plein » s'oppose à un Massif central « vide», etc. 

est nécessaire au message que l'on peut juger par ailleurs. Le problème n'est pas tant non plus que la France métropolitaine ne soit plus qu'une vaste surface d'aplanissement sans gradients climatiques est-ouest et nord-sud, cela fait partie des choix initiaux, de la posture retenue que l'on peut critiquer par ailleurs. Le problème est que cette représentation n'explique rien, même si on peut considérer qu'elle montre tout. Elle n'explique pas la poussée millénaire de l'Etat français, en gros, vers le nord-est (donc la forme de la frontière) tout comme elle n'explique pas par quels mécanismes théoriques s'est mis en place le réseau urbain fractal métropolitain, ni sa distribution spatiale par exemple. C'est la fameuse question du pourquoi là et pas ailleurs et dont la réponse ne peut pas être seulement : parce qu'ailleurs il y a autres choses, en jouant sur les interactions dans la mesure où ce type de raisonnement devient rapidement tautologique. Cette solution présente donc une objectivation de la représentation certes, mais qui fait écran à l'objectivation «faible» (car non théorisable) de l'histoire, et surtout ici à l'objectivation «forte " (car pouvant être théorique) à laquelle commencent à nous faire accéder les sciences de la complexité, en focalisant sur des symétries très spécifiques donc très partielles. En cela ce fut certainement une étape nécessaire. Elle doit être aujourd'hui dépassée.

41 L'exemple précédent est ancien en partie parce que l'hexagone comme figure euclidienne utilisée pour représenter la France métropolitaine est une pratique des écoles normales (communication orale de Christian Grataloup, 2004, Avignon, Groupe Dupont) et que le schéma chorématique proposé par R. Brunet a été publié en 1973. Cela étant on pourrait se demander s'il est daté. S'il correspond à une période et à une vision qui ont leurs avantages. Pour essayer d'aller plus loin il est possible de trouver un exemple de modèle graphique plus actuel (Picouet et Renard, 2002) pour montrer simplement que la prégnance de ces symétries galiléennes (translation, rotation, etc.) est toujours aussi forte. Le but n'étant évidemment pas, là non plus, de faire une critique des choix faits par ces collègues qui ont opté pour ce mode de représentation, ce qui est parfaitement légitime. Le but est d'attirer l'attention sur l'usage peut-être même subliminal qui est fait de la symétrie dans les représentations les plus récentes; ce qui encore une fois peut parfaitement se concevoir comme moyen de représentation et de communication mais qui pose un problème par exemple au regard de la fractalité du monde : focaliser sur des symétries très partielles peut masquer une symétrie bien plus globale et donc détourner d'une théorisation possible. Le problème est donc fondamentalement que le monde ne peut être décrit théoriquement par cette voie alors qu'elle pourrait le laisser penser.

L'approche est ici à plus grande échelle si on la compare à celle appliquée à l'hexagone français. La figure $\mathrm{n}^{\circ} 5$ montre très clairement qu'il y a une double symétrie de fait : au nord la Mer du Nord, au sud la Manche, à l'est la France, à l'ouest l'Angleterre. Le site étant une ancienne vallée submergée après la dernière glaciation. L'organisation nordsud du bras de mer s'en déduit. Ce dernier ayant, si ce n'est empêché, du moins fortement limité les possibilités d'expansion de l'un des deux Etats sur le territoire actuel de l'autre, du moins en gros depuis la fin de la guerre de Cent ans. Dans ces conditions il est «logique » de retrouver là une frontière internationale. Le détroit au sens strict correspondant à la rencontre de ces deux structures étatiques. Cette organisation peut d'ailleurs être décomposée et schématisée. 
Figure 6 : Modalités de construction d'un modèle graphique de détroit (d'après B. Picouet

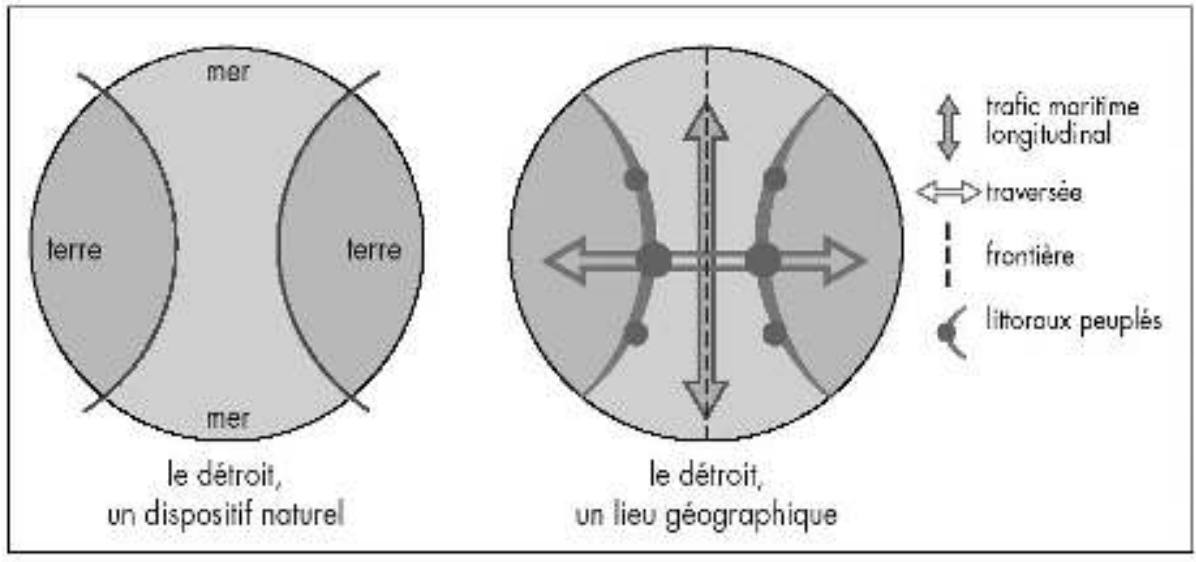

Les auteurs au bas de la figure nous disent que c'est un territoire dissymétrique. Qu'estce qu'il y a de dissymétrique, au moins dans la représentation, dans l'organisation spatiale du détroit du Pas de Calais, donnée par B. Picouet et J.-P. Renard (2002)? Plusieurs axes de symétries sont clairement portés sur ce schéma. Le pointillé vertical matérialise le rail qui ordonne le lieu et le sens de cheminement des bateaux indiqués par les deux flèches. Le tracé du tunnel sous la Manche et de ses prolongements tant en France qu'en Angleterre sépare une partie nord d'une partie sud du détroit. Même les côtes, à une petite différence près au nord, sont symétriques! A cela s'ajoute l'opposition symétrique des flux : par bateau selon un axe nord-sud, par train selon un axe ouest-est; selon un rail maritime montant et descendant; selon une circulation ferroviaire bi directionnelle dans le tunnel. L'ensemble constituant une croix qui pourrait pivoter sur son intersection (symétrie par rotation).

Reste le réseau urbain constitué de trois agglomérations de chaque côté (donc symétrique) mais avec - ce qui conduit certainement les auteurs à parler de dissymétrie - une différence dans la taille entre celles de l'Est, plus importantes et celles de l'Ouest, toujours de taille semblable. La dissymétrie de l'établissement urbain n'est donc pas niable d'autant qu'elle se double d'une hiérarchie en France qui n'apparaît pas en Angleterre mais cela ne doit pas conduire à occulter totalement la forte symétrie en miroir selon deux axes, du schéma. La perfection aurait conduit à avoir des villes dans un réseau hiérarchique de même taille des deux côtés. C'est d'ailleurs ce qui apparaît dans le modèle (figure 6).

Celui-ci est une généralisation de la situation du Pas de Calais. La représentation se fonde sur une structure spatiale de base, parfaitement symétrique et totalement épurée, constituée d'un disque où sont disposés symétriquement les différents éléments statiques du territoire. Ainsi qu'une représentation des flux. C'est un référentiel hautement symétrique à partir duquel un niveau de dissymétrie peut être établi.

\subsection{4. - -Une représentation qui rend euclidien ce qui ne l'est guère}

La réalisation fréquente de chorèmes ou de graphiques, voire même de schémas euclidiens en géographie, alors même que l'essentiel des exemples dont disposent les chercheurs est non euclidien ne concoure-t-elle pas à masquer la "véritable » nature de l'espace géographique? Tel est au fond le risque. Celui de s'inscrire dans une façon 
de penser, dans des catégories dont au final on se trouve prisonnier? Par ailleurs la géométrisation, c'est-à-dire largement la régularisation, des limites afin d'obtenir des formes euclidiennes s'apparente à une simplification évidente (un tracé lisse, régulier est plus facile à dessiner). Que perdons-nous en faisant cela dans la représentation certes mais aussi dans l'éventail des approches que nous réduisons fortement? Reste la question de la matérialité. Les processus de production d'ouvrages, de cartes, de représentations accessibles «au premier coup d'œil» intègrent difficilement l'irrégularité, c'est-à-dire une symétrie de nature fractale. Cela devrait peut-être conduire à développer d'autres approches comme des images véristes numériques qui pourraient avoir une profondeur scalaire. La prise en compte de la symétrie conduit donc aussi à une remise en cause de la cartographie au sens large.

\section{3. - Conclusion}

Dans le domaine scientifique, passées les premières surprises et interrogations, la symétrie par dilatation, la géométrie fractale, tout comme le chaos, ont été acceptés pour ce qu'ils sont. Cela ne semble pas être le cas sur un plan culturel plus général, d'autant que ces nouvelles conceptions ont des conséquences importantes dans la marche des sociétés. La symétrie apparait comme un élément essentiel de la relation entretenue entre l'homme occidental et sa "production intellectuelle». Il y a là une symétrie en miroir de type sujet/objet. On retrouve ainsi le dualisme grec. Par ailleurs pour se constituer, la science moderne a dû rompre les liens ancestraux entre l'humanité et la nature, entre l'homme et son environnement. En cela elle a instrumentalisé le dualisme sujet/objet et a cherché à objectiver des entités choisies et définies en fonction de projets. Or il se trouve aujourd'hui que cette structure de pensée est, pour le moins dans plusieurs domaines comme l'environnement au sens écologique du terme (réchauffement de la planète, etc.), problématique. Elle apparaît difficilement tenable en tant que telle car elle ne permet pas de réintroduire l'homme dans la nature. En d'autres termes il faut arrêter d'instrumentaliser la symétrie dans un dualisme binaire pour l'utiliser dans un formalisme théorique, pour s'en servir dans la construction d'une théorie géographique qui permette de dépasser la coupure entre géographie physique et géographie humaine.

Toutefois, si cette symétrie en miroir (en opposition) est culturelle, héritée des Grecs, comme le laisse penser le fait que la culture chinoise ne semble pas avoir développé la même perspective duale (symétrique) alors une modification de notre registre culturel sera nécessaire mais ne semble pas hors de portée. Si par contre il y a derrière cette façon de penser un support biologique contraignant fort, ce qui est peu probable, alors le travail à faire semble d'une toute autre ampleur. Cela étant, il faut bien voir ici que cette question de la symétrie ouvre sur plusieurs problèmes essentiels pour la géographie qui s'intègrent dans ce cadre. Il y a d'une part celui de savoir quelle est la nature profonde de la symétrie (dimension ontologique). Répondre à cette question a une conséquence forte sur la re-naturalisation de la géographie. Il y a d'autre part celui de l'articulation de la symétrie avec la conservation et la relativité (cf. le théorème d'E. Noether). Cela constitue la base ternaire fondamentale d'une théorisation en géographie. Il y a enfin la question des types de symétries et parmi ceux-ci celui de la symétrie déployée dans l'ordre des échelles (fractale) qui est plus apparente aujourd'hui mais qui n'est pas aussi cruciale qu'on pourrait le penser dans l'explication 
théorique fondamentale tout en étant, pour le géographe, un moyen théorique de description et de questionnement incontournable. C'est ce que nous avons essayé d'indiquer en rappelant quelques problèmes que pose la chorématique.

En conclusion nous espérons avoir montré que la question de la symétrie est un problème incontournable, qu'elle est particulièrement complexe et fondamentale, certes pour la physique ou les mathématiques, mais aussi pour la géographie. Ce texte qui n'est qu'une esquisse, ne fait qu'effleurer bien des problèmes qui demanderaient de longs développements. Nous espérons toutefois qu'il aura le mérite d'attirer l'attention de la communauté sur cette question en montrant son importance pour une géographie plus conceptuelle qu'elle ne l'est ou que nous aimerions qu'elle le soit.

\section{BIBLIOGRAPHIE}

BoIs E. (2002). - L'univers sans repos ou l'essence première du mouvement. Peter Lang éditeur, Berne, «Philosophia Naturalis et Geometricalis », 237 p.

Bouligand Y, Castellengo M., Cohen-Tannoudji G., Couder Y., Dagognet F., Dhombres J., Luminet J.P., Prochiantz A., de Ricqles A., Saunier J.-P., Thom R., Vidal C., Virilio P. et Yaguello M. (1994). Les sciences de la forme aujourd'hui, Seuil, Paris, «Point Sciences », 188 p.

BRUNET R. (1968). - Les phénomènes de discontinuité en géographie, Mémoires et documents du CNRS, année 1967, nouvelle série, vol. 7, Editions du CNRS, Paris, 111 p.

BRUNET R. (1972). - « Organisation de l'espace et cartographie de modèles : les villes du Massif central », L'espace géographique, $\mathrm{n}^{\circ} 1, \mathrm{p}$. 43-48.

BRUNET R. (1973). - « Structure et dynamique de l'espace français : schéma d'un système », L'espace géographique, $\mathrm{n}^{\circ}$ 2, p. 249-255.

BRUNET R. (2001). - Le déchiffrement du monde. Théorie et pratique de la géographie. Belin, Paris, $401 \mathrm{p}$.

D'ARCY THOMPSON (1942), Ongrowth and form. The complete revised edition, réimpression Dover, publications Inc, New York, 1116 p. ; $1^{\text {re }}$ édition 1917.

D'ARCY THOMPSON (1994). - Forme et croissance, Seuil, édition du CNRS, Paris, 335 p.

DeLANNOY J.-J., (1997). - Recherches géomorphologiques sur les massifs karstiques du Vercors et de la transversale de Ronda - Andalousie - Espagne. Les apports morphogéniques du karst. Thèse d'État Université de Grenoble, Éditions du Septentrion, 687 p.

FORRIEZ M. et MARTIN Ph. (2006). - « De l'utilité de la théorie de la relativité d'échelles de L. Nottale en géographie. Recherche d'un modèle scalaire spatio-temporel ", Géopoint 2006 : demain la géographie, Avignon, Groupe Dupont et UMR ESPACE 6012 du CNRS éditeurs, Brouillon Dupont, p. 63-64 et : http://www.groupe-dupont.org

FORRIEZ M. et MARTIN Ph. (à paraître). - De l'utilité de la théorie de la relativité d'échelles de L. Nottale en géographie. Partie II : Application d'un modèle scalaire spatio-temporel. Géopoint 2006 : demain la géographie, Avignon, Groupe Dupont et UMR ESPACE 6012 du CNRS éditeurs, 9 p. 
HUBERT J. P. (1993). - La discontinuité critique. Essai sur les principes a priori de la géographie humaine, Publications de la Sorbonne, Paris, $221 \mathrm{p}$.

LоснAк G. (1994). - La géométrisation de la physique. Flammarion éditeur, Paris, coll. : Champs, $272 \mathrm{p}$.

MARTIN Ph. (2000.a). - Quelle est la dimension du massif karstique de la Sainte Baume ? Eléments pour une théorie spatiale et fractale du karst. Karstologia, n 35, p. 13-26.

MARTIN Ph. (2000.b). - Forme et rugosité des surfaces karstiques. Conséquences pour une théorie spatiale et fractale de l'interface terrestre. Karstologia, $\mathrm{n}^{\circ}$ 36, p. 1-16.

MARTIN Ph. (2003). - Objectivation des formes en géographie et calculs d'indicateurs fractals. Exemples karstiques. In : Objets et indicateurs géographiques sous la direction de J. MABY, Collection Actes Avignon n 5, Université d'Avignon et UMR ESPACE 6012 du CNRS éditeurs, p. 153-268, 13 fig., 1 graphe. Texte en ligne sur les sites : http://www.umrespace.org et http://www.geo.univavignon.fr.

MARTIN Ph. (2004). - Modélisation fractale et structurelle des formes en géographie. Réflexion développée à partir d'exemples karstiques. Habilitation à diriger les recherches. Université d'Avignon et des Pays du Vaucluse, tome I, 173 p., tome II, 314 p., tome III, 176 p., 1 carte coul. ht.

MARTIN Ph. (2006.a). - La forme peut-elle libérer la prisonnière du carrefour ? Vers une théorie de la forme en géographie. Colloque Géopoint 2004 : La forme en géographie, Avignon, Groupe Dupont et UMR ESPACE 6012 du CNRS éditeurs, p. 19-38.

MARTIN Ph. (2006.b). - Caractérisation fractale du relief « stationnarisé » du Lodévois. Colloque SAGEO 2006, Cdrom ISBN : 2-9526014-1-0, Laboratoire : Image et ville et Université L. Pasteur, Strasbourg éditeur, $23 \mathrm{p}$.

MARTIN Ph. et ForRIEZ M. (à paraître). - De l'utilité de la théorie de la relativité d'échelles de L. Nottale en géographie. Partie I : Recherche d'un modèle scalaire spatio-temporel. Géopoint 2006 : Demain la géographie, Avignon, Groupe Dupont et UMR ESPACE 6012 du CNRS éditeurs, 6 p.

NOTTALE L. (1993). — Fractal space-time and microphysics. Towards a theory of scale relativity. Word Scientific Publishing, London, 333 p.

NotTALE L. (1998). - La relativité dans tous ses états. Hachette, Paris, 319 p.

PicouET P. et RENARD J. P. (2002). - Les détroits : de nouveaux territoires ? L'exemple du pas de Calais. Mappemonde, $\mathrm{n}^{\circ} 1,65$, p. 7-11.

STEVENS P.-S. (1978). - Les formes dans la nature, Seuil, Paris, 232 p.

STEWART I. (1982). - Les chroniques de Rose Polymath : Oh catastrophe. Belin diffuseur, 70 p.

Tном R. (1973). - La science malgré tout... In : Encyclopaedia Universalis, Vol. 17, Organum, Paris, p. 5-10.

Tном R. (1977). - Stabilité structure et morphogenèse. $2^{\mathrm{e}}$ édition, InterEditions, Paris, 351 p. ; $1^{\text {re }}$ édition anglaise : 1972 .

Tном R. (2003). - CEuvres complètes. CD-Rom, Institut des Hautes Etudes Scientifiques, Bures-surYvette, éditeur. 


\section{RÉSUMÉS}

La symétrie est basée sur l'idée platonicienne d'harmonie. Comprise comme un élément d'une plus grande complexité, c'est aujourd'hui un principe scientifique fondamental qui interpelle notre discipline et l'invite à reformuler un ancrage théorique. L'espace géographique ne peut être par définition un espace asymétrique (sans symétrie), car il ne possèderait aucune manifestation traduisant son organisation (espace homogène et isotrope). En quoi intervient alors la symétrie? Les translations (mais aussi les rotations et surtout les dispositifs en miroir) sont souvent considérées, en géographie, comme correspondant seules au concept de symétrie alors que celle-ci apparaît aussi fondamentalement dans les manifestations fractales tangibles (formes) ou structurelles (lois). Associé à des formes euclidiennes, cet aspect très partiel de la symétrie constitue un élément essentiel de la pratique chorématique. Nous craignons qu'une telle approche (associant perception et représentation) fasse obstacle (obstruction) à une théorisation formelle de l'espace géographique qui l'instrumentaliserait.

A symmetry is based on the Platonic idea of harmony. It is a fundamental scientific principle today. The geographic space cannot be by definition an asymmetric space (without symmetry). In that case, this space would possess no demonstration translating its organization (homogeneous and isotropic space). The translations, (but also the rotations and especially the devices in mirror) are often considered, in geography, as the concept of the symmetry while this one also appears in the fractal forms and in the structural laws. Associated to an euclidian forms this partial aspect of the symmetry constitutes an essential element of the practice of the chorématique. We are afraid that such an approach (associating perception and representation) establishes an obstacle (obstruction) to a formal theorization of the geographic space which uses the symmetry.

Die Symmetrie findet ihren Sinn mit der altgriechischen Idee der Harmonie. In einer tieferen Bedeutung dient sie heute als ein zentraler Begriff für verschiedenen Naturwissenchaften. Eine solche Annahme fordert die Geographie dazu auf, seine theoretische Grundlagen neu zu interpretieren. In der Tat kann der geographische Raum nicht als a-symmetrisch (d.h. homogen und isotrop) verstanden werden, sonst würde seine geographische Grundlage verneint. Man darf aber der Begriff nicht auf $\mathrm{zu}$ visuellen, unklaren, und sogar betrüglichen Vorstellungen beschränken (wie z.B. die Chorematik um Roger Brunet es meint), mit der Symmetrie ist auch die geometrische Interpretation der fraktalen Dimension zu verstehen, die der Geographie zu ganz neuen theoritischen Einheitsperpektiven einführen wird.

\section{INDEX}

Mots-clés : Fractale, chorème, dissymétrie, morphogenèse, espace

Schlüsselwörter : Fraktal, Chorème, Asymmetrie, Morphogenese, geographischer Raum

Keywords : Fractal, chorème, dissymmetry, morphogenesis, space

\section{AUTEUR}

\section{PHILIPPE MARTIN}

Université d'Avignon, UMR ESPACE 6012 du CNRS, 74 rue Pasteur, Case 17, 84029 Avignon CEDEX 1.

philippe.martin@univ-avignon.fr 